\title{
RECOVERY AND UTILIZATION OF OLD SLLK DEGUMMING BATHS
}

\author{
By Risaku Tsunokae. \\ (Received Apr.9th., 1926)
}

I. In spite of the long history of boiling-off of the silk, it was only towards the last decade of the past century that man felt it mas necessary to recover and reutilize the old silk degumming baths. The much increase in the price of soap, and the difficulty of conducting the boiling-off operation at a sufficiently low cost to compensate for the higher cost of labour, invited man to this consideration.

II. Boiled-off liquor is now employed in the dyeing of silk, as it is found to have good levelling or equalizing properties, by causing the coloring matter to go more slowly on the fibre.

III. The composition of the boiled-off liquor is much liable to variation as the silk scourers differ from one another in the quantity of soap to be used in the treatment of raw silk. The constituents of value in the liquor are, however, no other than the fatty matter and the sericine or silk gum, in every case.

IV. A sample of boiled-off liquor, examined by the author, sho weda speçific gravity of 1.010 , and contained:

$\begin{array}{ll}\text { Soap } & 1.119 \% \\ \text { Free fatty acids } & 0.143 \\ \text { Sericine } & 0.316\end{array}$

V. Some reagents such as sulphate of alumina, sulphate of copper, lead acetate and chloride of calcium, precipitate both the soap and silk gum as curdy mass. This can be separated through filtration from the quite clear waterly liquid which is in a good condition for running off. The mass of lead soap and sericine thus obtained contains the largest quantity of the silk gum of all, the next is that of aluminium soap, and that of copper soap comes the third.

VI. The mass of copper soap and silk gum, which is precipitated by adding the sulphate of copper into the boiling-off liquor, and separated through filteration, can be elmulsified by the treatment with such emulsifiers as Turkeyred oil, gum arabic, potassium soap etc.. The emulsified copper soap is very good fungicide and has a great demand from gardners and pomologists. The method has been patented for the author. (Japanese Patent No. 65055)

VII. The mass of metallic soap and silk gum thus obtained is mixed with a small quantity of water and strong acid such as hydrochloric acid or 
sulphuric acid, the mixture of fatty acid and silk gum separates and collects on the surface of the aqueous liquid, and it can be easily skimmed off. The fatty matter can be separated from the sericine by the special steam distillation method, invented by the author, which enables the distillation at by far the lower temperature than the usual method. In this special distillation method, the author uses not only the vapour of the steam, but also utilizes that of the toluene, xylene, or other solvents immiscible to water and having lower boiling points than those of the fatty acids. By this process the distillation temp. of the fatty acid can be depressed about $30-50^{\circ} \mathrm{C}$ than those of the original ones.

\title{
ON THE SCROOP OF THE NATURAL SILK AND THE WAY TO PRODUCE IT ON THE RAYON
} (ARTIFICIAL SILK)

\author{
By Risaku Tsunokae.
}

(Received March 13th., 1926)

Preface

When the scoured silk fibre is squeezed or pressed it makes a crackling sound which is termed as "Scroop."

Several explanations to account for the scroop have been given, and these can be classified into two groups : the one is that the scroop of the silk is an inherent property of the fibre itself and it is produced by the scouring of it. But if the silk is badly treated in the course of degumming and aftertreatments, the scroop will be lost, but it may acquire again when it is worked in a bath of dilute acids. The other explanation is that the scroop of silk is not the original property of its fibre but an acquired one in the boiling-off operation and other aftertreatments.

On artifcial silks it is generally supposed that the scroop can not be produced on its fibre, but some writer says it can be done by passing the fibre through a soap bath at first and then through a bath containing a small quantity of acid, and drying it without further washing. According to the author's investigations, the crackling sound which can be termed generally as "Scroop" is not the inherent property of the natural silk fibre, but it is a 